\title{
Effect of Adrenergic Drugs on the Lipopolysaccharide-In- duced Oxidative Stress and Liver Damage in the Rat
}

\author{
Omar ME Abdel Salam, ${ }^{1 *}$ Amany Ameen Sleem, ${ }^{2}$ Rehab Fawzy Abdel Rahman, ${ }^{2}$ Fatma A Morsy ${ }^{3}$ \\ ${ }^{1}$ Department of Toxicology and Narcotics, National Research Centre, Cairo, Egypt \\ ${ }^{2}$ Department of Pharmacology, National Research Centre, Cairo, Egypt \\ ${ }^{3}$ Department of Pathology, National Research Centre, Cairo, Egypt
}

Abstract

Aim: We aimed to investigate the effect of the adrenergic system on oxidative stress and liver damage during mild systemic inflammation in rats.

Methods: For this purpose, Eschrechia coli lipopolysacchride (LPS; $300 \mu \mathrm{g} / \mathrm{kg}$ ) was intraperitoneally injected either alone or along with the $\alpha-2$ adrenoceptor antagonist yohimbine, the non-selective $\beta$ adrenoceptor antagonist propranolol, the and $\beta$-adrenoceptor agonist adrenaline and rats euthanized $4 \mathrm{~h}$ later. The effects of these drugs in saline-treated rats were also studied. Serum alanine aminotransferase (ALT), aspartate aminotransferase, and alkaline phosphatase activities were measured. Liver tissue levels of malondialdehyde (MDA), nitric oxide (NO), reduced glutathione (GSH) were determined and liver histology evaluated.

Results: In saline-treated rats (i) yohimbine caused significantly increased ALT, AST and ALP in serum. In addition, yohimbine increased MDA, NO and decreased GSH in liver tissue and caused cytoplasmic vacuolations and cellular infiltration: (ii) adrenaline treatment increased serum ALT, AST, liver MDA, decreased liver GSH and caused minute vacuolar degeneration and foci of necrosis; (iii) in contrast, there were no biochemical or histologic alterations after propranolol treatment. In endotoxaemic rats; (i) serum aminotransferases and alkaline phosphatase were significantly increased by yohimbine together with increased liver MDA, NO and decreased GSH content; (ii) serum ALT decreased by propranolol and adrenaline while serum ALP increased by adrenaline; (iii) liver GSH increased by propranolol; (iv) LPS administration caused acute liver damage in the form of foci of necrosis, vacuolar degeneration and aggregates of inflammatory cellular infiltration. Compared with the LPS only group, treatment with yohimbine increased while propranolol or adrenaline produced less liver damage.

Conclusions: Thus, blockade of $\alpha-2$ adrenoceptors increased while $\beta$ adrenoceptor antagonism decreased oxidative stress and liver damage following LPS administration in rats. Collectively, these results indicate a benefit from blockade of beta-adrenoceptors in protecting the liver during mild systemic inflammation in part by decreasing oxidative stress.

Keywords: Catecholamines, Lipopolysaccharide endotoxin, Oxidant stress, Hepatic injury, Adrenergic receptors, Yohimbine, $\beta$ Adrenoceptor antagonist

\section{Introduction}

Lipopolysaccharide, a structural component of the outer wall of Gram-ve bacteria, is the cause of septic shock and multi-organ failure. ${ }^{1}$ LPS is recognized by Toll-like receptor 4 (TLR4), a member of transmembrane pattern recognition receptors on the outer membrane of immune cells, the activation of which acts to induce an innate immune response and inflammation. Consequent to their stimulation, immune cells eg., macrophages and neutrophils induces the activation of the transcription factor nuclear factor kappa-B (NF- $\mathrm{B}$ )) and the cellular expression of genes encoding proinflammatory cytokines such as tumour necrosis factor (TNF)- $\alpha$, inter-
Quick Response Code:

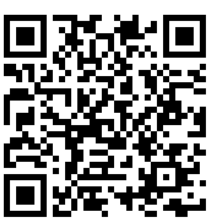

*Corresponding author: Omar ME Abdel Salam, Department of Toxicology and Narcotics, National Research Centre, Tahrir Street, Dokki, Cairo, Egypt

Received: 29 July, 2021

Published: 16 August, 2021

Citation: Abdel Salam OME, Ameen Sleem A, Abdel Rahman RF, Morsy FA. Effect of Adrenergic Drugs on the Lipopolysaccharide-Induced Oxidative Stress and Liver Damage in the Rat. SOJ Diabet Endocr Care. 2021;1(2):1-10. DOI: 10.53902/SOJDEC.2021.01.000507 
leukin-1 $\beta$ (IL-1 $\beta$ ) and IL-6, monocyte chemoattractant protein, cell adhesion molecules, with resultant systemic inflammatory syndrome manifested by tissue injury, increased vascular permeability, and, ultimately, multiple organ failure and shock. ${ }^{2,3}$ The peripheral administration of LPS in rodents induces the release of proinflammatory cytokines, lipid mediators of inflammation, reactive oxygen species (ROS) and nitric oxide both in periphery and brain. ${ }^{4,5}$ TLR4 is expressed by hepatocytes, Kupffer cells, hepatic stellate cells, sinusoidal endothelial cells and dendritic cells in the liver. LPS exerts hepatic injury by stimulating inflammatory cells and hepatic Kupffer cells, the resident macrophages of the liver, to produce various inflammatory cytokines and interferon-gamma (IFN- $\gamma)^{6,7}$ TNF- $\alpha$ is a major determinant of liver injury. It induces activation of caspases and apoptosis in hepatocytes prior to secondary necrosis and release of aminotransferases by activation of TNF receptor- $1{ }^{8}$ Recruited neutrophils and Kupffer cells in liver sinusoids also produce large amounts of reactive oxygen species (ROS) e.g. $\mathrm{H}_{2} \mathrm{O}_{2}$ and superoxide anion, causing oxidative stress which is also an important contributor to hepatocyte death via mechanisms that involve mitochondrial dysfunction, inhibit mitochondrial respiration and ATP depletion. ${ }^{9,10}$

There is evidence for a neural reflex control of acute inflammation. Thus, there exists a cholinergic anti-inflammatory mechanism whereby vagus nerve efferent's by the action of acetylcholine on nicotinic receptors on tissue macrophages act to inhibit the expression and release of TNF, IL-1, HMGB1 and other cytokines from macrophages. ${ }^{11,12}$ On the other hand, the role of the adrenergic system in modulating the levels of cytokines and tissue damage during end toxaemia is less clear. For example, either stimulation of $\alpha-2$ adrenoceptors or their blockade was reported to attenuate tissue damage caused by LPS. ${ }^{13-15}$ In addition, chemical sympathectomy attenuated the LPS-induced increase in plasma cytokines. ${ }^{16}$ while bilateral sectioning of the greater splanchnic sympathetic nerves increased TNF- $\alpha$ levels in response to LPS. ${ }^{17}$

The purpose of this study was therefore to examine the effect of adrenergic drugs on oxidative stress and liver damage during mild systemic inflammation.

\section{Materials and Methods}

\section{Animals}

Male Sprague-Dawley rats obtained from the Animal House Colony of the National Research Centre (NRC), weighing 150-160g of body weight were used in the study. Standard laboratory food and water were provided ad libitum. Animal procedures followed the recommendations of the Ethics Committee of the NRC and of the National Institutes of Health Guide for Care and Use of Laboratory Animals (Publication No. 85-23, revised 1985). Equal groups of six rats each were used in all experiments.

\section{Drugs and chemicals}

A purified lyophilized Escherichia coli end toxin (Serotype 055:B5) purchased from Sigma Company (St Louis, MO, USA) was used and dissolved in sterile saline, aliquoted, and frozen at $-20^{\circ} \mathrm{C}$. Other drugs used were propranolol hydrochloride, yohimbine hydrochloride (Sigma USA), and adrenaline (Nile Pharmaceutical Co., Egypt). The remaining chemicals and reagents were of analytical grade and obtained from Sigma (Sigma, USA).

\section{Experimental groups}

Rats were randomly assigned to different treatment groups (6rats/group) as follows:

Group 1 was treated with the vehicle (saline) and served as -ve control.

Group 2 was treated with LPS intraperitoneally (i.p.) at a dose of $300 \mu \mathrm{g} / \mathrm{kg}$ to induce endotoxemia and served as a +ve control.

Group 3 received i.p. injection of propranolol at a dose of $2 \mathrm{mg} /$ $\mathrm{kg}$.

Group 4 was treated with LPS and propranolol ( $2 \mathrm{mg} / \mathrm{kg}$, i.p.).

Group 5 was treated with yohimbine at a dose of $4 \mathrm{mg} / \mathrm{kg}$, i.p.

Group 6 received i.p. injection of LPS+yohimbine ( $4 \mathrm{mg} / \mathrm{kg}$, i.p).

Group 7 was treated with adrenaline at a dose of $1 \mathrm{mg} / \mathrm{kg}$, i.p.

Group 8 received i.p. injection of LPS+adrenaline (1mg/kg, i.p).

These drugs were given at the same time as LPS-challenge. Four hours after LPS injection, blood samples were taken from the orbital venous plexus under light ether anaesthesia, from which clear sera were obtained. Sera were used for the assessment of alanine aminotransferase (ALT), aspartate aminotransferase (AST), and alkaline phosphatase (ALP) activities. Rats were then euthanized by decapitation. Livers were collected and parts of the tissues were preserved in $10 \%$ formalin for further histopathological examination. Other parts were preserved at $-80^{\circ} \mathrm{C}$ for further determination of malondialdehyde (MDA), reduced glutathione (GSH), and nitric oxide in tissues homogenate.

\section{Biochemical assessments}

Oxidative stress biomarkers

Liver oxidative stress biomarkers were assessed in homogenates by determining malondialdehyde (MDA), reduced glutathione (GSH) and nitric oxide (NO). MDA, an end product of lipid peroxidation was determined by measuring thiobarbituric reactive substances (TBAS) using the method by Nair and Turne ${ }^{18}$ in which TBAS reacts with thiobarbituric acid forming TBA-MDA adduct and the absorbance is read at $532 \mathrm{~nm}$ using spectrophotometer. GSH was determined using Ellman's reagent [DTNB (5,5'-dithiobis (2-nitro- 
benzoic acid)] which is reduced by the free sulfhydryl group on the GSH molecule generating 5-thio-2-nitrobenzoic acid. The latter has yellow colour and can be determined by reading absorbance at $412 \mathrm{~nm} .{ }^{19} \mathrm{NO}$ estimated as nitrate/nitrite was determined by the method of Miranda et al $^{20}$

\section{Serum liver enzymes}

Serum activities of alanine aminotransferase (ALT) and aspartate aminotransferase (AST) were determined according to Reitman-Frankel colorimetric transaminase procedure..$^{21}$ using commercially available kits from BioMérieux (France).

\section{Liver histology}

Liver tissues were fixed in freshly prepared 10\% neutral buffered formalin, processed routinely, and embedded in paraffin. Liver sections $(5 \mu \mathrm{m})$ were stained with hematoxylin-eosin and examined by light microscope.

\section{Statistical analysis}

All data were expressed as the mean \pm SEM. Data were analyzed by one-way analysis of variance (ANOVA) followed by followed by Tukey's multiple comparisons test. GraphPad Prism 6 for Windows (GraphPad Prism Software Inc., San Diego, CA, USA) was used. Statistical significance was considered at a probability value of less than 0.05 .

\section{Results}

\section{Oxidative stress}

\section{Saline-treated rats}

Rats treated with yohimbine exhibited significantly increased MDA by $21.8 \%$ (26.8 \pm 1.02 vs. $22.0 \pm 0.98 \mathrm{nmol} /$ g.tissue) and NO by $32.4 \%$ ( $63.03 \pm 4.2$ vs. $47.6 \pm 2.5 \mu \mathrm{mol} /$ g.tissue) compared to the saline control group. Meanwhile, GSH in liver tissue showed significant decrease by $35.7 \%$ ( $3.87 \pm 0.24$ vs. $6.02 \pm 0.38 \mu \mathrm{mol} / g$.tissue $)$ after yohimbine administration. Significantly increased liver MDA by $36.4 \%$ ( $30.0 \pm 1.48$ vs. $22.0 \pm 0.98 \mathrm{nmol} /$ g.tissue), and decreased GSH by $37.4 \%$ ( $3.77 \pm 013$ vs. $6.02 \pm 0.38 \mu \mathrm{mol} / \mathrm{g}$.tissue) were also observed in adrenaline treated rats compared to saline control. The values for MDA, NO and GSH in the propranolol treated group were not different from the saline group.

\section{LPS-treated rats}

LPS administration induced a significant increase in MDA by $64.5 \%$ ( $36.2 \pm 0.88$ vs. $22.0 \pm 0.98 \mathrm{nmol} /$ g.tissue) and NO levels by $46.7 \%$ (69.83 \pm 3.2 vs. $47.6 \pm 2.5 \mu \mathrm{mol} /$ g.tissue) whereas GSH decreased by $53.0 \%$ ( $2.83 \pm 017$ vs. $6.02 \pm 0.38 \mu \mathrm{mol} / g$.tissue $)$ compared to the saline control group. The LPS/yohimbine group exhibited significantly higher MDA, NO and lower GSH values compared to the LPS only group. MDA increased by $34.0 \%$ (48.5 \pm 2.34 vs. $36.2 \pm 0.88 \mathrm{nmol} /$ g.tissue) and NO increased by $20.6 \%(84.23 \pm 4.0$ vs. $69.83 \pm 3.2 \mu \mathrm{mol} /$ g.tissue), while GSH decreased by $17.7 \%$ (2.33 \pm 0.14 vs. $2.83 \pm 017 \mu \mathrm{mol} / g$.tissue) compared to LPS control. In the LPS/adrenaline group no significant differences were observed as regards MDA, and GSH compared to the LPS control. The level of NO, however, significantly decreased by $19.1 \%$ (56.47 \pm 3.1 vs. $69.83 \pm 3.2 \mu \mathrm{mol} / \mathrm{g}$.tissue) after adrenaline treatment. In contrast, liver GSH increased by propranolol by $40.3 \%$ compared to the LPS control value ( $3.97 \pm 0.17$ vs. $2.83 \pm 017 \mu \mathrm{mol} /$ g.tissue).

\section{Serum enzymes}

\section{Saline-treated rats}

The treatment of rats with yohimbine caused significantly increased activities of ALT, AST and ALP in serum compared to the saline control group. Serum ALT and AST increased by $29.7 \%$ (79.3 \pm 1.5 vs. $61.13 \pm 2.27 \mathrm{U} / \mathrm{l}$ ) and $15.7 \%$ ( $88.83 \pm 2.4$ vs. $76.75 \pm 2.22$ U/l) while ALP activity was increased by $27.3 \%$ (175.1 \pm 7.91 vs $137.6 \pm 6.53 \mathrm{U} / \mathrm{l})$. The treatment of rats with adrenaline increased serum ALT by $17.9 \%$ (72.1 \pm 2.14 vs. $61.13 \pm 2.27 \mathrm{U} / \mathrm{l})$ and AST by $33.4 \%$ (102.4 \pm 3.66 vs. $76.75 \pm 2.22 \mathrm{U} / \mathrm{l}$ ) compared to the saline control.

\section{LPS-treated rats}

After LPS challenge the serum enzyme activities of ALT and AST activities increased significantly by $69.0 \%$ (103.3 \pm 4.52 vs. $61.13 \pm 2.27 \mathrm{U} / \mathrm{l})$ and $74.1 \%$ (133.6 \pm 8.8 vs. $76.75 \pm 2.22 \mathrm{U} / \mathrm{l})$ in LPS treated rats compared to control saline group. Meanwhile, serum ALP was significantly increased by $95.1 \%$ by LPS injection (268.4 \pm 6.7 vs. $137.6 \pm 6.53 \mathrm{U} / \mathrm{l})$. Serum aminotransferases and ALP were significantly increased by yohimbine. Compared to the LPS only control, serum ALT and AST activities were increased by $17.0 \%(120.9 \pm 3.8$ vs. $103.3 \pm 4.52 \mathrm{U} / \mathrm{l})$ and $22.7 \%(163.9 \pm 7.53$ vs. $133.6 \pm 8.8 \mathrm{U} / \mathrm{l}$ ) in the LPS/yohimbine treated group. Serum ALP was also increased by $43.3 \%$ ( $384.5 \pm 12.3$ vs. $268.4 \pm 6.7 \mathrm{U} / \mathrm{l}$ ) following yohimbine treatment. In contrast, serum ALT was significantly decreased by propranolol and adrenaline by $24.4 \%$ and $16.6 \%$ from control value of $103.3 \pm 4.52$ to $78.08 \pm 3.1$ and $86.1 \pm 4.0 \mathrm{U} / \mathrm{l}$, respectively. On the other hand, significantly increased serum ALP activity by $60 \%$ was observed in the LPS/adrenaline treated group compared to the LPS control ( $429.2 \pm 13.1$ vs. $268.4 \pm 6.7 \mathrm{U} / \mathrm{l})$. (Figure 1 \& Figure 2)

\section{Histological results of liver}

The liver of normal rats revealed normal characteristic hepatic architecture (Figure 3A). The liver of rats treated with LPS only showed dilated, congested portal vein and cellular infiltration around. Aggregate area of cellular infiltration and foci of necrosis were seen (Figure 3B, Figure 3C, Figure 3D \& Figure 3E). The liver from rats treated with yohimbine only showed severe cytoplasm vacuolations, dilated, and congested portal vein and cellular infiltration around (Figure 4A). Liver sections from rats treated with 
LPS along with yohimbine showed dilated and congested portal tract, dilated bile duct and massive cellular infiltration were seen.
Minute vacuolar degeneration and cellular infiltration in sinusoidal space could be observed (Figure 4B \& Figure 4C).
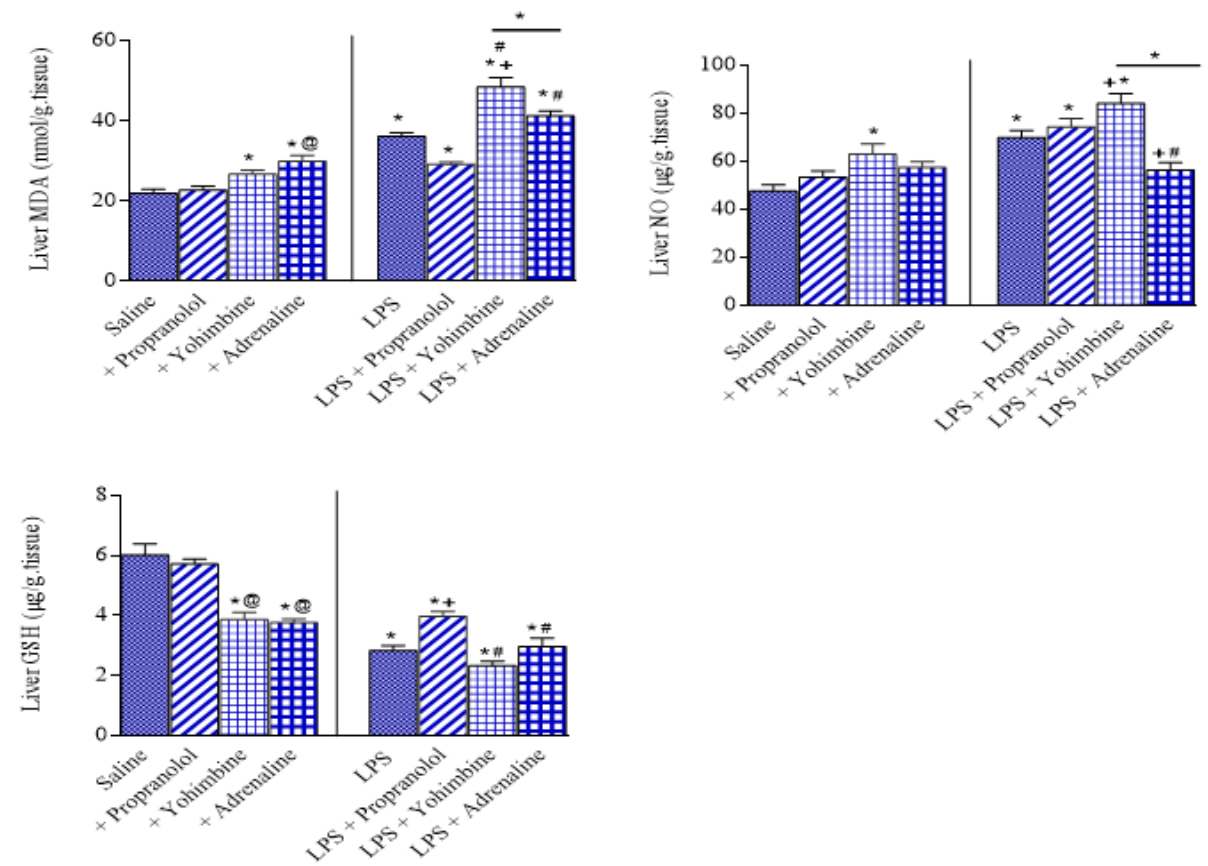

Figure 1: Effect of propranolol, yohimbine or adrenaline on liver malondialdehyde (MDA), nitric oxide (NO) and reduced glutathione (GSH) in LPS-treated rats. ${ }^{*} \mathrm{p}<0.05$ vs. saline and between different groups as shown on the graph. @: $\mathrm{p}<0.05$ vs. saline/propranolol. +: $p<0.05$ vs. LPS control. \#: $\mathrm{p}<0.05$ vs. IPS/propranolol.
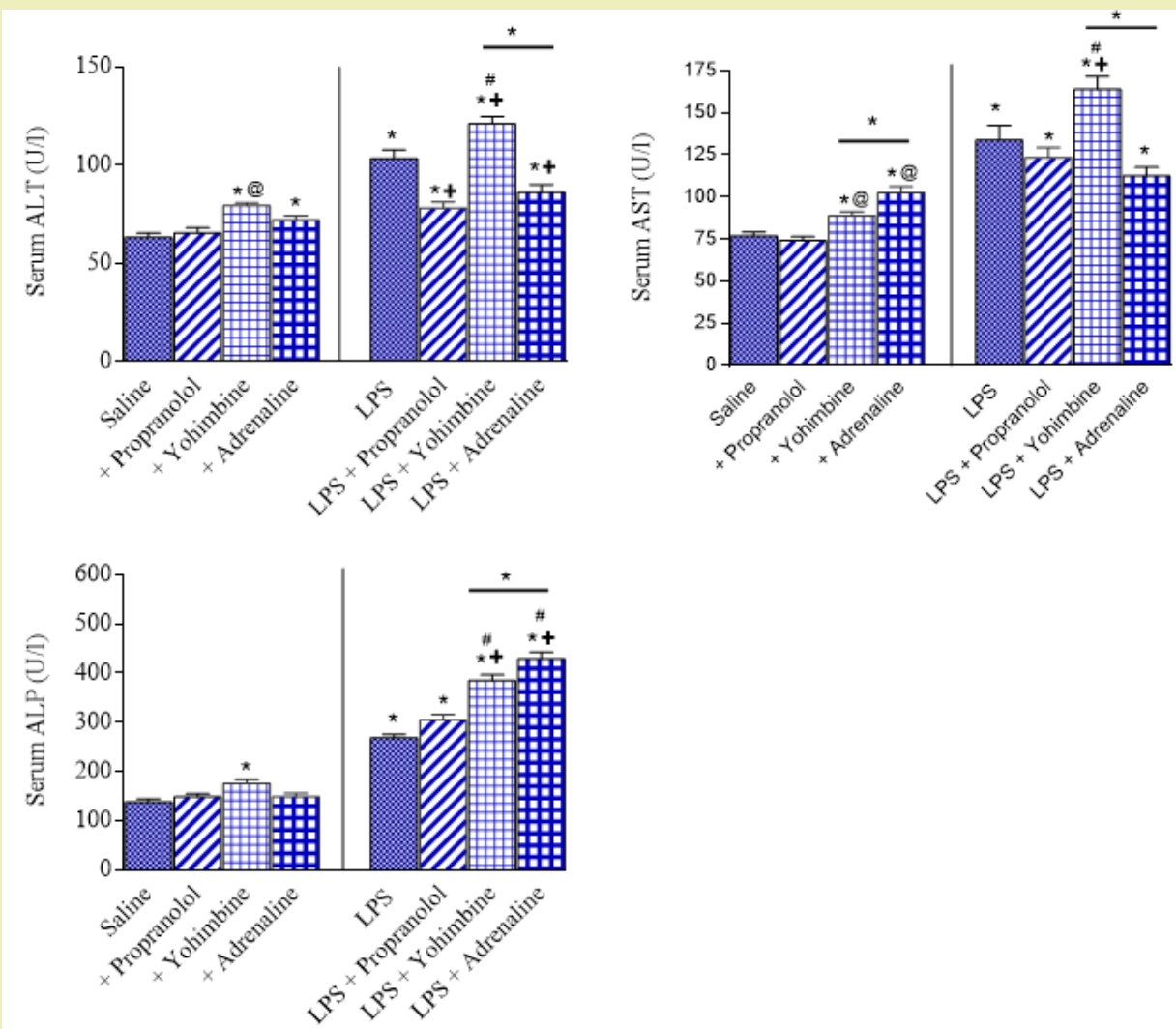

Figure 2: Effect of propranolol, yohimbine or adrenaline on serum alanine aminotransferase (ALT), aspartate aminotransferase (AST) and alkaline phosphatase (ALP) in LPS-treated rats. *p<0.05 vs. saline and between different groups as shown on the graph. @: p $<0.05$ vs. saline/propranolol. +: p $<0.05$ vs. LPS control. \#: p<0.05 vs. IPS/propranolol. 

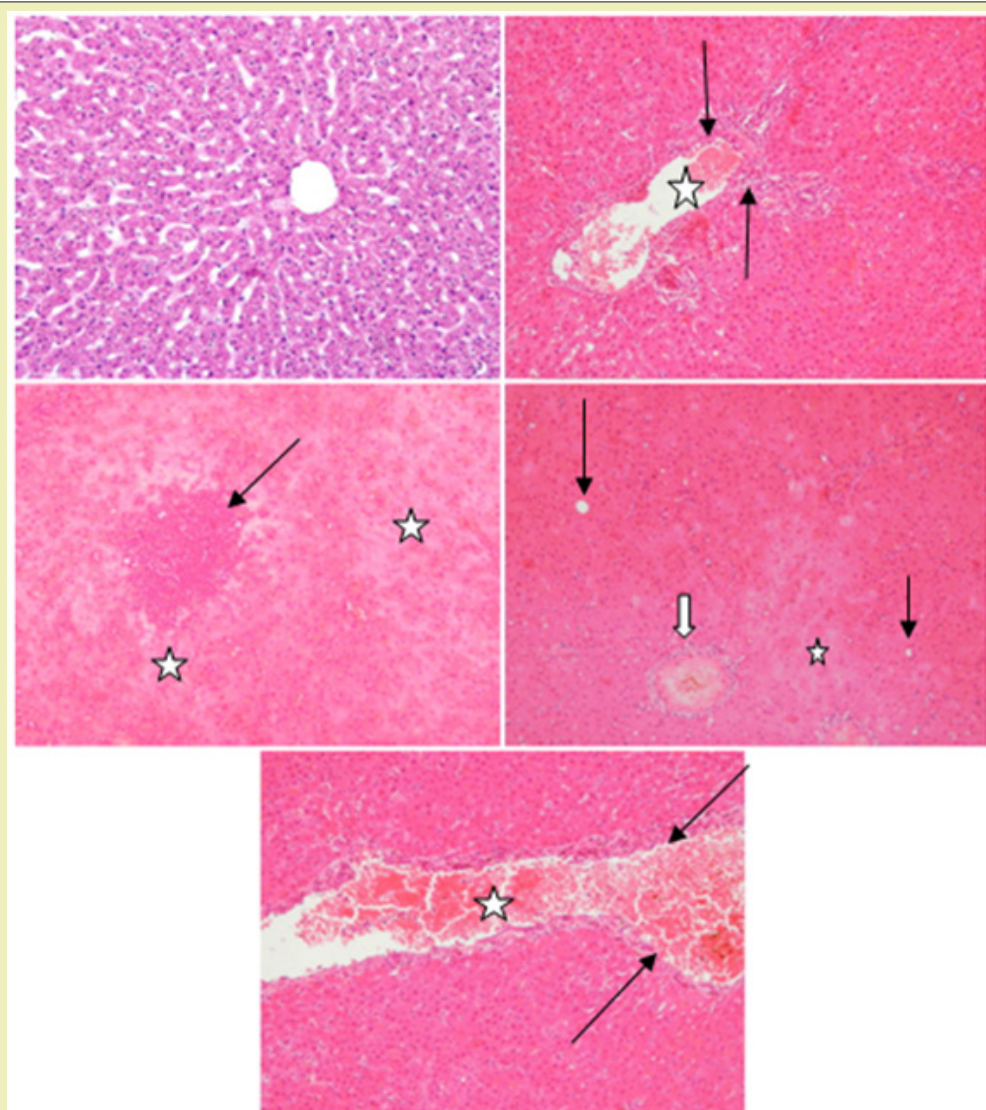

Figure 3: H\&E staining of the liver after treatment with: (A) Saline: normal hepatic architecture. (B) LPS only: dilated, congested portal vein (star) and cellular infiltration around (arrow). (C) LPS only: aggregate area of cellular infiltration (arrow), foci of necrosis, edema in liver tissue (star), destruction with sinusoidal architecture. (D) LPS only: minute vacuolar degeneration (arrow), dilated and congested central vein (white arrow). (E) LPS only: dilated, congested portal vein (star) and cellular infiltration around (arrow) (Hx\&Ex100).

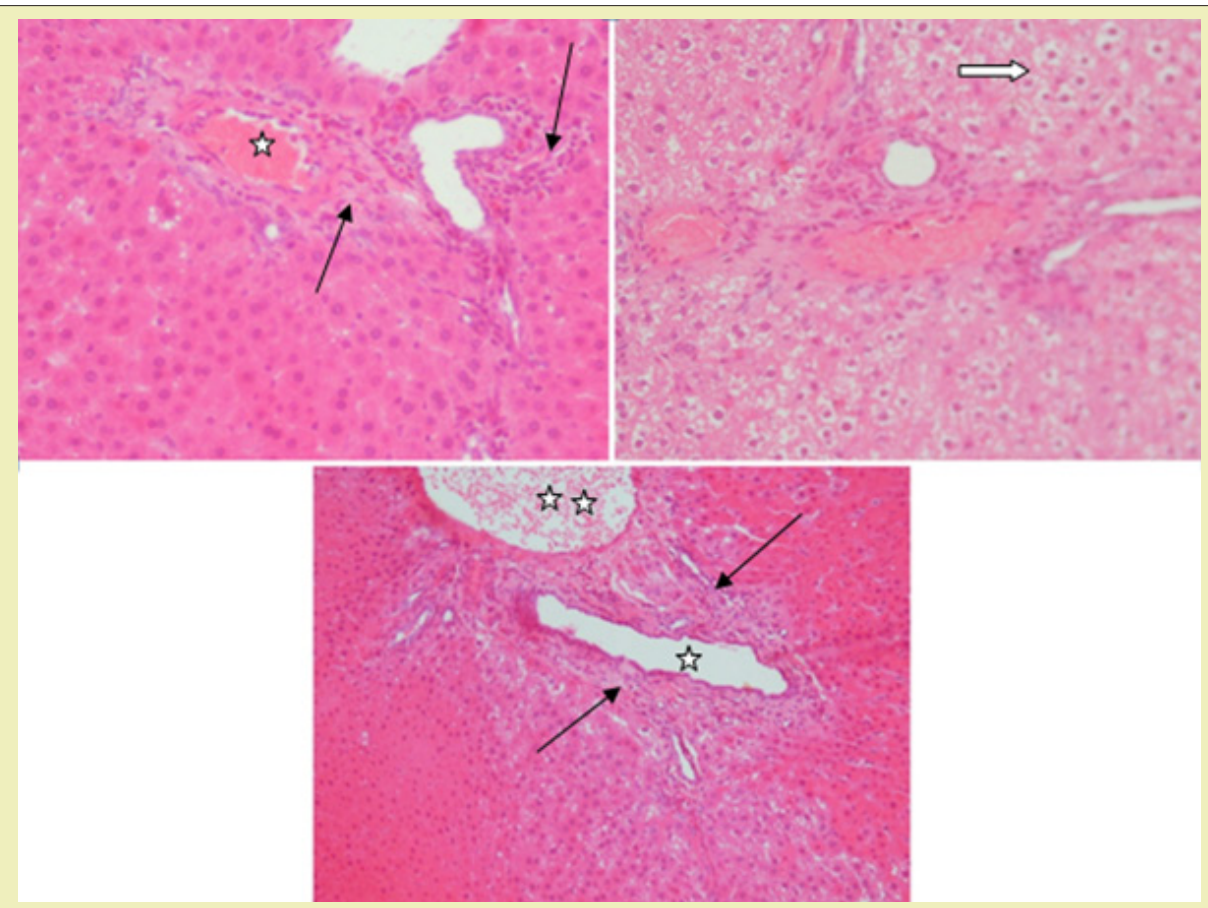

Figure 4: H\&E staining of the liver after treatment with: (A) Yohimbine only: dilated and congested of portal vein (star) and cellular infiltration around (arrow) (Hx\&Ex200). (B) LPS+yohimbine: severe cytoplamic vacuolation (white arrow) (Hx\&E 200). (E) LPS+yohimbine (another field): dilated and congested portal tract with thickening of portal vein vascular wall, fibrotic (two stars), dilated bile duct (star) and massive cellular infiltration (arrow), (Hx\&Ex200). 
Rats treated with adrenaline only exhibited dilated and congested central vein, cellular infiltration around and in sinusoidal space. Minute vacuolar degeneration and foci of necrosis could be observed. Sings of degeneration in the form of pyknosis and karyolysis were noticed (Figure 5A \& Figure B). Rats treated with LPS and adrenaline showed improvement in pathological changes compared with the group treated with LPS only in the form of normal hepatic architecture but dilated, congested portal vein and dilated bile duct could be observed. Minute vacuolar degeneration and cellular infiltration around, dilated and congested central vein were seen. Foci of necrosis and karyolysis of some hepatocytes could be observed (Figure 5C, Figure 5D, Figure 5E \& Figure 5F).

No pathological changes could be observed in the case of rats treated with propranolol only (Figure 6A \& Figure 6B). Rats treated with LPS and propranolol showed some improvement in pathological changes in the form of no massive areas of necrosis, but foci of necrosis, hepatocytes appeared with variable size and some exhibited karyolysis (Figure 6C \& Figure 6D).

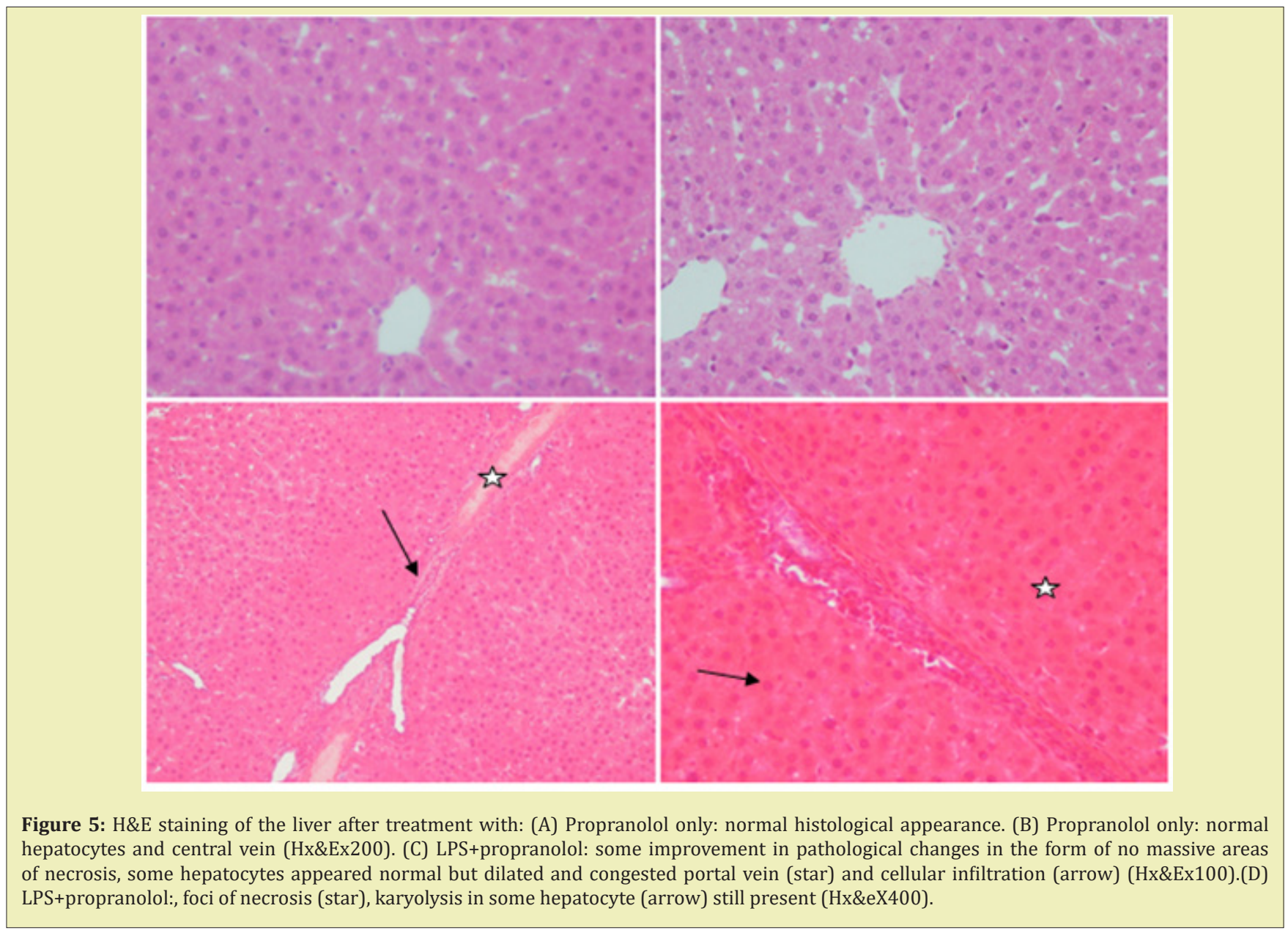

\section{Discussion}

In this study, we attempt to delineate the role of adrenergic mechanisms on the development of liver tissue damage during mild systemic inflammation. We administered the $\alpha-2$ adrenoceptor antagonist yohimbine, the non-selective $\beta$ adrenoceptor antagonist propranolol the and $\beta$-adrenoceptor agonist adrenaline under basal conditions and during LPS endotoxaemia. Our findings can be summarized as follows: (i) in saline-treated rats, yohimbine and adrenaline were associated with the development of liver damage; (ii) propranolol did not induce any pathological changes in saline-treated rats; (iii) after LPS injection, liver damage was increased by yohimbine with increased serum liver enzymes and oxidative stress; (iv) LPS-induced liver damage showed some improvement by propranolol (together with reduced release of aminotransferases, and oxidative stress) and also by adrenaline.

After LPS injection, a significant rise in the level of the lipid peroxidation end product malondialdehyde was observed in liver tissue, indicative of an increase in ROS and consequent attack on membrane lipids. Meanwhile, the level of the antioxidant molecule and free radical scavenger reduced glutathione (GSH) fell which might be due to inhibition of biosynthesis and/or failure of replenishing the GSH stores during systemic inflammation. There was also significant increase in liver nitric oxide by LPS which is consistent with previous studies in which LPS was i.p. given at doses of 200- 
$300 \mu \mathrm{g} / \mathrm{kg} .{ }^{22,23}$ In response to LPS the inducible form of nitric oxide synthase (iNOS) is rapidly upregulated within hours in hepatocytes and in resident hepatic macrophages (Kupffer cells). ${ }^{24}$ iNOS releases excessive amounts of nitric oxide for longer time which is asso- ciated with cell injury. ${ }^{24-26}$ Our histopathological study showed that LPS induced necrotic foci, hypertrophy of Kupffer cells, sings of degeneration dilated, congested blood vessels and cellular infiltration which is in agreement with previous studies. ${ }^{5,22}$

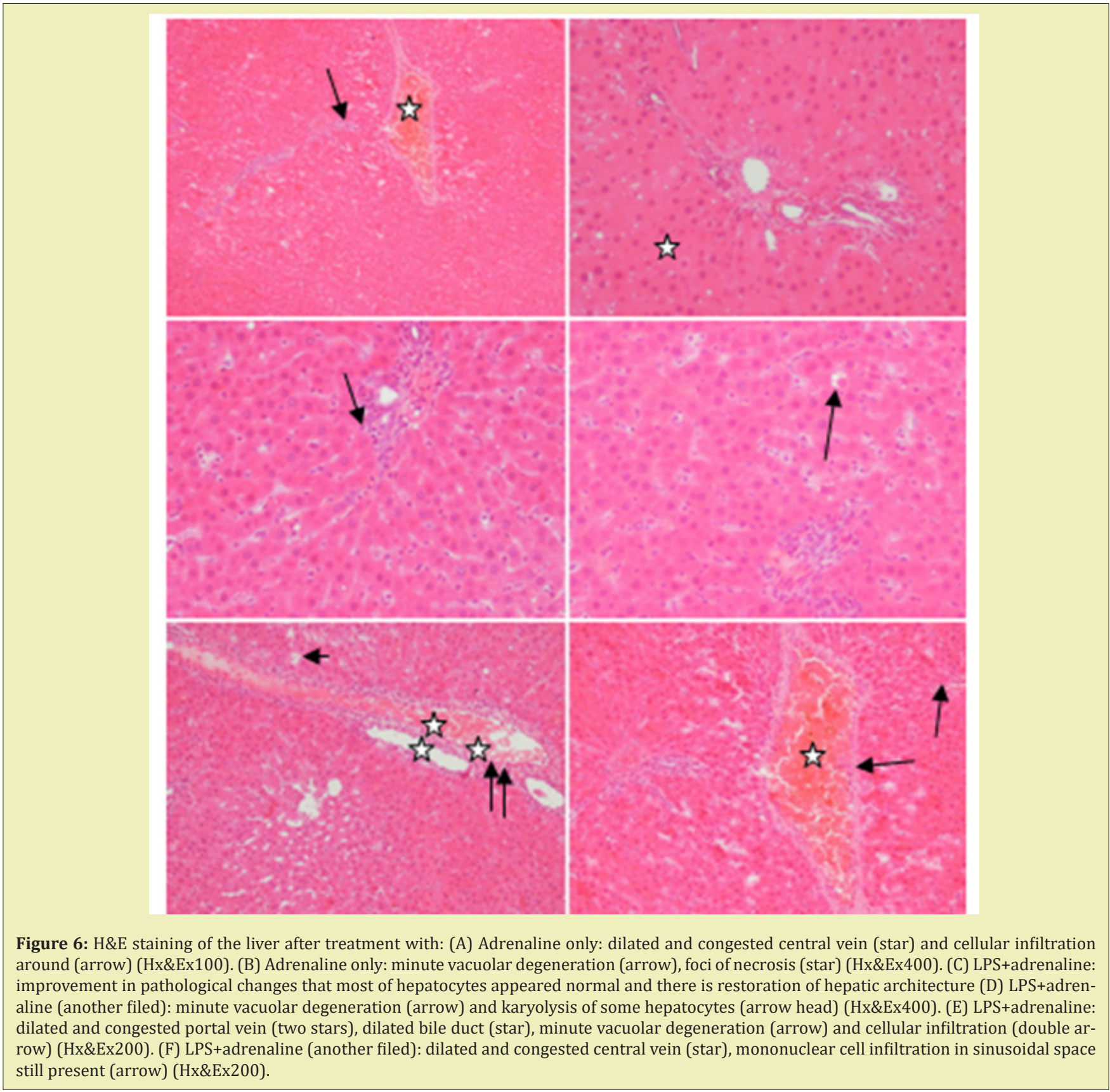

Yohimbine, an indole alkaloid extracted from the bark of the Pausinystalia yohimbe tree, is a potent selective antagonist at $\alpha 2$-adrenoceptors that produces adrenergic activation via an action on presynaptic $\alpha 2$-adrenergic autoreceptors. ${ }^{27-29}$ We showed that yohimbine resulted in an increase in serum aminotransferases in both saline and LPS-treated rats and increased lipid peroxidation in endotoxaemia. The drug induced severe cytoplasmic vacuolation, dilated, congested portal vein and cellular infiltration in saline-treat-

ed rats. When given to LPS-treated rats, yohimbine did not change the histopathological picture, and even massive inflammatory cellular infiltration was observed. Other researchers also reported enhancement of LPS-induced liver injury in rats given yohimbine $(250 \mu \mathrm{g} / \mathrm{kg}$, i.p.) prior to endotoxin administration with marked increments in lipid peroxidation, proinflammatory cytokines and amino transferases in plasma and liver tissue and increased histological damage. ${ }^{13}$ In contrast, yohimbine given at a dose of $2 \mathrm{mg} / \mathrm{kg}$ 
prior to restraint stress decreased ALT and AST activities in plasma and apoptosis (caspase activation in hepatocytes). ${ }^{30} \alpha 2$-adrenoceptors are widely distributed in the central nervous system and periphery and modulate noradrenergic nervous system outflow from the brain. Their inhibition by yohimbine increases central sympathetic outflow. ${ }^{29}$ The drug also increases venous plasma and tissue concentrations of noradrenaline. ${ }^{27}$ Yohimbine by increasing sympathetic tone and nor adrenaline release and indirectly resulting in vasoconstriction is thus likely to exacerbate the toxin-induced liver damage.

The catecholamine's adrenaline and noradrenalin are widely used in clinical practice in the management of septic shock in Gram -ve septicaemia to restore adequate blood pressure and tissue perfusion. ${ }^{31}$ Our results showed that adrenaline, an $\alpha$ and $\beta$-adrenergic agonist, given to saline treated rats induced minute vacuolar degeneration and foci of necrosis as well as dilated and congested central vein and cellular infiltration. There was however, some improvement in histopathological changes in endotoxaemic rats given adrenaline compared to their controls. In these rats, adrenaline treatment decreased serum ALT and AST but increased ALP. In their study, von Montfort et al. ${ }^{32}$ demonstrated that adrenaline enhanced LPS-induced liver damage with some necro-inflammatory foci and enhanced neutrophil infiltration. In LPS-treated rats, adrenaline also increased plasma AST levels. In vitro experiments suggested that adrenaline is able to modulate the immune response to LPS. In human mononuclear cells stimulated with LPS, adrenaline induced IL-10 synthesis while suppressing TNF- $\alpha .^{33}$ It increased LPS-stimulated IL-8 production in human whole blood via $\beta$-adrenergic receptors. ${ }^{34}$ Rough et $a .^{35}$ however, reported that adrenaline pretreatment significantly increased LPS-induced TNF- $\alpha$ production in RAW 264.7 cells. Adrenaline was also reported to suppress nitric oxide production by LPS-stimulated macrophages. ${ }^{36}$ By virtue of its effects on the synthesis of inflammatory cytokines, chemokines and nitric oxide; adrenaline might thus be able to modulate the extent of organ damage during endotoxaemia. Stimulation of the sympathetic supply to blood vessels of the liver, however, will result in vasoconstriction and reduced blood flow. ${ }^{37}$ Pure $\beta$-adrenergic agonists dilate hepatic arterioles, and increase hepatic artery flow in contrast to pure $\alpha$-adrenergic agonists that reduce blood flow through the hepatic artery. ${ }^{38}$ Clearly, the effect of adrenaline on liver injury during endotoxaemia would be the net result of both $\alpha$ - and $\beta$-adrenoceptor stimulation on the vasculature and the inflammatory response.

Our results show in addition that rats treated with LPS/propranolol exhibited less liver damage compared with the group treated with only LPS. The drug reduced serum ALT activity, liver MDA and increased liver GSH in endotoxaemic rats. Several studies have focused on the role of adrenoceptors in endotoxaemia but with controversial results being reported. It has been shown that $\beta$-adrenoceptor stimulation modulates the inflammatory response during end toxemia. For example, terbutaline was reported to suppress the increase in plasma TNF- $\alpha$ and IL- 6 and TNF- $\alpha$ mRNA in kidney, spleen and thymus after LPS injection in rats; effects that were blocked by propranolol. ${ }^{39}$ In lung tissue, isoproterenol reduced TNF- $\alpha$ production and lipid peroxidation induced by LPS. ${ }^{40}$ Protective effects were also reported for the $\beta 2$-adrenoceptor agonist clenbuterol in liver tissue as opposed to propranolol $(25 \mu \mathrm{g} /$ $\mathrm{kg}$ ) which increased damage in clenbuterol/LPS-treated rats. ${ }^{41} \mathrm{On}$ the other hand, pretreatment with the selective $\beta 1$-adrenoceptor blockers atenolol and metoprolol conferred protection against lethal endotoxemia in rats. $\beta 1$ blockade decreased hepatic expression of proinflammatory cytokines and plasma IL-6. ${ }^{42}$ In RAW 264.7 cells, $\beta 2$ blockade attenuated LPS stimulated TNF-alpha production. ${ }^{35}$ Moreover, Özyllmaz et al. $^{43}$ reported beneficial effects for propranolol $(10 \mathrm{mg} / \mathrm{kg}$ ) pretreatment on endothelial lung injury in sepsis. Other researchers provided data that in mice with operative injury, propranolol significantly decreased TNF- $\alpha$ and IL-6 production from splenic macrophages stimulated with LPS. ${ }^{35}$ The administration of propranolol also attenuated the ethanol-induced sensitization of LPS-induced liver damage. ${ }^{32}$ It has been suggested that in sepsis, $\beta 2$ activation as well as $\beta 1$ blockade down regulate proinflammatory response and improve the alterations in immune, cardiovascular and coagulation systems. ${ }^{44}$ Evidence for a beneficial effect from beta adrenoceptor blockade in sepsis also derives from several randomized clinical trials and systematic reviews that demonstrated favorable effects for the use of beta blockers on heart rate without decrementing blood pressure and reduced mortality. ${ }^{45-49}$ For example, In patients with severe sepsis, Gadallah et al. reported 28-day mortality and ICU stay and control of heart rate after treatment with the beta blocker esmolol. ${ }^{46}$ It has also been shown that combining both metoprolol and milrinone led to improved hemodynamic in patients with sepsis. ${ }^{48}$ Our results support these studies and do suggest a hepatoprotective effect for propranolol during mild systemic inflammation. Rats treated with LPS and propranolol did not show massive areas of necrosis as their LPS only counterparts.

In conclusion, in an experimental model of mild systemic inflammatory illness caused by i.p. injection of LPS end toxin, the concurrent administration of the beta adrenoceptor blocker propranolol was associated with reduced liver injury. This effect involved at least in part decreased hepatic oxidative stress.

\section{Strength and limitation of the study}

The study focused on oxidative stress as a principal mechanism underlying liver tissue injury and the biochemical results were confirmed by the histological study which added strength to the work. Inflammatory mediators, were however, not assessed. 


\section{Future research perspectives}

Further research is necessary to delineate the benefit of using beta blockers in sepsis, the underlying mechanisms as well as differences between clinically used beta-blockers.

\section{Acknowledgments}

None.

\section{Funding}

This work was not supported by research grants.

\section{Conflicts of Interest}

The authors declare no conflicts of interest.

\section{References}

1. Alexander C, Rietschel ET. Bacterial lipopolysaccharides and innate immunity. J Endotoxin Res. 2001;7(3):167-202.

2. Decker T. Sepsis: avoiding its deadly toll. JClin Invest. 2004;113(10):13871389.

3. Stearns Kurosawa DJ, Osuchowski MF, Valentine C, et al. The Pathogenesis of Sepsis. Annu Rev Pathol. 2011;6:19-48.

4. Qin L, Wu X, Block ML, et al. Systemic LPS causes chronic neuroinflammation and progressive neurodegeneration. Glia. 2007;55(5):453-462.

5. Abdel Salam OME, Sleem AA, Youness ER, et al. Neuro- and hepatoprotective effects of methylene blue in rats treated with lipopolysaccharide endotoxin. Reactive Oxygen Species. 2018;6(17):325-337.

6. Malhi H, Gores GJ. Cellular and molecular mechanisms of liver injury. Gastroenterology. 2008;134(6):1641-1165.

7. McAvoy EF, Mc Donald B, Parsons SA, et al. The role of CD14 in neutrophil recruitment within the liver microcirculation during endotoxemia. J Immunol. 2011;186(4):2592-2601.

8. Wullaert A, van Loo G, Heyninck K, et al. Hepatic tumor necrosis factor signalling and nuclear factor-kappa B: effects on liver homeostasis and beyond. Endocr Rev. 2007;28(4):365-386.

9. Jones BE, Lo CR, Liu H, et al. Role of caspases and NF-kappaB signaling in hydrogen peroxide- and superoxide-induced hepatocyte apoptosis. Am J Physiol Gastrointest Liver Physiol. 2000;278(5):G693-G699.

10. Wu D, Cederbaum AI. Alcohol, oxidative stress, and free radical damage. Alcohol Res Health. 2003;27(4):277-284.

11. Tracey KJ. The inflammatory reflex. Nature. 2002;420(6917):853-859.

12. Pavlov VA, Wang H, Czura CJ, et al. The Cholinergic anti-inflammatory pathway: A missing link in neuroimmunomodulation. Mol Med. 2003;9(5-8):125-134.

13. Fessler HE, Otterbein L, Chung HS, et al. Alpha-2 adrenoceptor blockade protects rats against lipopolysaccharide. Am J Respir Crit Care Med. 1996;154(6 Pt 1):1689-1693.

14. Chen JH, Yu GF, Jin SY, et al. Activation of $\alpha 2$ adrenoceptor attenuates lipopolysaccharide-induced hepatic injury. Int J Clin Exp Pathol. 2015;8(9):10752-10759.

15. Shimokawa T, Yoneda $\mathrm{K}$, Yamagata $\mathrm{M}$, et al. Yohimbine ameliorates lipopolysaccharide-induced acute kidney injury in rats. Eur J Pharmacol. 2020;871:172917.

16. Mravec B. Chemical sympathectomy attenuates lipopolysaccharide-induced increase of plasma cytokine levels in rats pretreated by ACTH. $J$ Neuroimmunol. 2019;337:577086.

17. Martelli D, Yao ST, McKinley MJ, et al. Neural control of inflammation by the greater splanchnic nerves. Temperature (Austin). 2014;71(1):1415 .
18. Nair V, Turner GA. The thiobarbituric acid test for lipid peroxidation structure of the adduct with malondialdehyde. Lipids. 1984;19:804-805.

19. Ellman GL. Tissue sulfhydryl groups. Arch Biochem Biophys. 1959;82(1):70-77.

20. Miranda KM, Espey MG, Wink DA. A rapid, simple spectrophotometric method for simultaneous detection of nitrate and nitrite. Nitric Oxide. 2001;5(1):62-71.

21. Crowley LV. The Reitman-Frankel colorimetric transaminase procedure in suspected myocardial infarction. Clin Chem. 1967;13(6):482487.

22. Abdel Salam OME, Youness ER, Mohammed NA, et al. Effect of ket amine on oxidative stress following lipopolysaccharide administration. Comp Clin Pathol. 2015;24(1):53-63.

23. Abdel Salam OME, Youness ER, Omara EA, et al. Effect of adipose tissue-derived mesenchymal stem cell treatmenton oxidative stress and inflammatory response following Escherichia coli lipopolysaccharide. Comp Clin Pathol. 2015;24:343-358.

24. Li J, Billiar TR. Nitric Oxide. IV. Determinants of nitric oxide protection and toxicity in liver. Am J Physiol (Gastrointest. Liver Physiol). 1999;276(5):G1069-G1073.

25. Zhang C, Walker LM, Hinson JA, et al. Oxidant stress in rat liver after lipopolysaccharide administration: effect of inducible nitric-oxide synthase inhibition. J Pharmacol Exp Ther. 2000;293(3):968-972.

26. La Mura V, Pasarin M, Rodriguez Vilarrupla A, et al. Liver sinusoidal endothelial dysfunction after LPS administration: a role for inducible-nitric oxide synthase. J Hepatol. 2014;61(6):1321-1327.

27. Hedner T, Edgar B, Edvinsson L, et al. Yohimbine pharmacokinetics and interaction with the sympathetic nervous system in normal volunteers. Eur J Clin Pharmacol. 1992;43(6):651-656.

28. Grossman E, Rosenthal T, Peleg E, et al. Oral yohimbine increases blood pressure and sympathetic nervous outflow in hypertensive patients. J Cardiovasc Pharmacol. 1993;22(1):22-26.

29. Tam SW, Worcel M, Wyllie M. Yohimbine: a clinical review. Pharmacol Ther. 2001;91(3):215-243.

30. Zhu Q, Gu L, Wang Y, et al. The role of alpha-1 and alpha-2 adrenoceptors in restraint stress-induced liver injury in mice. PLOS ONE 2014;9(3):e92125.

31. Myburgh JA. An appraisal of selection and use of catecholamines in septic shock-old becomes new again. Crit Care Resusc. 2006;8(4):353360.

32. von Montfort C, Beier J, Guo L et al. Contribution of the sympathetic hormone epinephrine to the sensitizing effect of ethanol on LPS-induced liver damage in mice. Am J Physiol Gastrointest Liver Physiol. 2008;294(5):G1227-G1234.

33. Siegmund B, Eigler A, Hartmann G, et al. Adrenaline enhances LPS-induced IL-10 synthesis: evidence for protein kinase A-mediated pathway. Int J Immunopharmacol. 1998;20(1-3):57-69.

34. van der Poll T, Lowry SF. Lipopolysaccharide-induced interleukin 8 production by human whole blood is enhanced by epinephrine and in hibited by hydrocortisone. Infect Immun. 1997;65(6):2378-2381.

35. Rough J, Engdahl R, Opperman K, et al. Beta2 Adrenoreceptor blockade attenuates the hyperinflammatory response induced by traumatic injury. Surgery. 2009;145(2):235-242.

36. Zinyama RB, Bancroft GB, Sigola LB. Adrenaline suppression of the macrophage nitric oxide response to lipopolysaccharide is associated with differential regulation of tumour necrosis factor- $\alpha$ and interleukin-10. Immunology. 2001;104(4):439-446.

37. Kurosawa M, Unno T, Aikawa $\mathrm{Y}$, et al. Neural regulation of hepatic blood flow in rats: an in vivo study. Neurosci Lett. 2002;321(3):145-148.

38. Gelman S, Mushlin PS. Catecholamine-induced changes in the splanchnic circulation affecting systemic hemodynamics. Anesthesiology. 2004;100(2):434-439. 
39. Nakamura A, Imaizumi A, Yanagawa Y, et al. Beta(2)-Adrenoceptor activation attenuates endotoxin-induced acute renal failure. J Am Soc Nephrol. 2004;15(2):316-325.

40. Zhang H, Kim YK, Govindarajan A, et al. Effect of adrenoreceptors on endotoxin-induced cytokines and lipid peroxidation in lung explants. Am J Respir Crit Care Med. 1999;160(5 Pt 1):1703-1710.

41. Izeboud CA, Hoebe KHN, Grootendorst AF, et al. Endotoxin-induced liver damage in rats is minimized by beta 2 -adrenoceptor stimulation. Inflamm Res. 2004;53(3):93-99.

42. Ackland GL, Yao ST, Rudiger A, et al. Cardioprotection, attenuated sys temic inflammation, and survival benefit of betal-adrenoceptor blockade in severe sepsis in rats. Crit Care Med. 2010;38(2):388-394.

43. ÖzyılmazE, Büyüknacar HSG, Bağır E, et al. Early propranolol treat ment ameliorates endothelial dysfunction in experimental septic lung. Adv Clin Exp Med. 2019;28(3):291-297.

44. de Montmollin E, AboabJ, Mansart A, et al. Bench-to-bedside review: Beta-adrenergic modulation in sepsis. Crit Care. 2009;13(5):230.
45. Lee YR, Seth MS, Soney D, et al. Benefits of beta-blockade in sepsis and septic shock: A systematic review. Clin Drug Investig. 2019;39(5):429_ 440.

46. Gadallah RR, Aboseif EMK, Ibrahim DA, et al. Evaluation of the safety and efficacy of beta blockers in septic patients: a randomized control trial. Ain-Shams Journal of Anesthesiology. 2020;12:57.

47. Hasegawa D, Sato R, Nishida O. $\beta 1$-blocker in sepsis. Journal of Intensive Care. 2021;9:39.

48. Sanfilippo F, Santonocito C, Morelli A, et al. Beta-blocker use in severe sepsis and septic shock: a systematic review. Curr Med Res Opin. 2015;31(10):1817-1825.

49. Li J, Sun W, Guo Y, et al. Prognosis of $\beta$-adrenergic blockade therapy on septic shock and sepsis: A systematic review and meta-analysis of randomized controlled studies. Cytokine. 2020;126:154916. 